\title{
A BASE-POINT-FREE DEFINITION OF THE LEFSCHETZ INVARIANT
}

\author{
VESTA COUFAL
}

Received 30 November 2004; Accepted 21 July 2005

In classical Lefschetz-Nielsen theory, one defines the Lefschetz invariant $L(f)$ of an endomorphism $f$ of a manifold $M$. The definition depends on the fundamental group of $M$, and hence on choosing a base point $* \in M$ and a base path from $*$ to $f(*)$. At times, it is inconvenient or impossible to make these choices. In this paper, we use the fundamental groupoid to define a base-point-free version of the Lefschetz invariant.

Copyright () 2006 Vesta Coufal. This is an open access article distributed under the Creative Commons Attribution License, which permits unrestricted use, distribution, and reproduction in any medium, provided the original work is properly cited.

\section{Introduction}

In classical Lefschetz fixed point theory [3], one considers an endomorphism $f: M \rightarrow M$ of a compact, connected polyhedron $M$. Lefschetz used an elementary trace construction to define the Lefschetz invariant $L(f) \in \mathbb{Z}$. The Hopf-Lefschetz theorem states that if $L(f) \neq 0$, then every map homotopic to $f$ has a fixed point. The converse is false. However, a converse can be achieved by strengthening the invariant. To begin, one chooses a base point $*$ of $M$ and a base path $\tau$ from $*$ to $f(*)$. Then, using the fundamental group and an advanced trace construction one defines a Lefschetz-Nielsen invariant $L(f, *, \tau)$, which is an element of a zero-dimensional Hochschild homology group [4]. Wecken proved that when $M$ is a compact manifold of dimension $n>2, L(f, *, \tau)=0$ if and only if $f$ is homotopic to a map with no fixed points.

We wish to extend Lefschetz-Nielsen theory to a family of manifolds and endomorphisms, that is, a smooth fiber bundle $p: E \rightarrow B$ together with a map $f: E \rightarrow E$ such that $p=p \circ f$. One problem with extending the definitions comes from choosing base points in the fibers, that is, a section $s$ of $p$, and the fact that $f$ is not necessarily fiber homotopic to a map which fixes the base points (as is the case for a single path connected space and a single endomorphism.) To avoid this difficulty, we reformulate the classical definitions of the Lefschetz-Nielsen invariant by employing a trace construction over the fundamental groupoid, rather than the fundamental group. 
In Section 2, we describe the classical (strengthened) Lefschetz-Nielsen invariant following the treatment given by Geoghegan [4] (see also Jiang [6], Brown [3] and Lück [8]). We also introduce the Hattori-Stallings trace, which will replace the usual trace in the construction of the algebraic invariant.

In Section 3, we develop the background necessary to explain our base-point-free definitions. This includes the general theory of groupoids and modules over ringoids, as well as our version of the Hattori-Stallings trace.

In Section 4, we present our base-point-free definitions of the Lefschetz-Nielsen invariant, and show that they are equivalent to the classical definitions.

\section{The classical theory}

2.1. The geometric invariant. In this section, $M^{n}$ is a compact, connected manifold of dimension $n$, and $f: M \rightarrow M$ is a continuous endomorphism.

The concatenation of two paths $\alpha: I \rightarrow X$ and $\beta: I \rightarrow X$ such that $\alpha(1)=\beta(0)$ is defined by

$$
\alpha \cdot \beta(t)= \begin{cases}\alpha(2 t) & \text { if } 0 \leq t \leq \frac{1}{2}, \\ \beta(2 t-1) & \text { if } \frac{1}{2} \leq t \leq 1 .\end{cases}
$$

The fixed point set of $f$ is

$$
\operatorname{Fix}(f)=\{x \in M \mid f(x)=x\} .
$$

Note that $\operatorname{Fix}(f)$ is compact. Define an equivalence relation $\sim$ on $\operatorname{Fix}(f)$ by letting $x \sim y$ if there is a path $v$ in $M$ from $x$ to $y$ such that $v \cdot(f \circ v)^{-1}$ is homotopic to a constant path.

Choose a base point $* \in M$ and a base path $\tau$ from $*$ to $f(*)$. Let $\pi=\pi_{1}(M, *)$. Given these choices, $f$ induces a homomorphism

$$
\phi: \pi \longrightarrow \pi
$$

defined by

$$
\phi([w])=\left[\tau \cdot(f \circ w) \cdot \tau^{-1}\right]
$$

where $[w]$ is the homotopy class of a path $w$ rel endpoints. Define an equivalence relation on $\pi$ by saying $g, h \in \pi$ are equivalent if there is some $w \in \pi$ such that $h=w g \phi(w)^{-1}$. The equivalence classes are called semiconjugacy classes; denote the set of semiconjugacy classes by $\pi_{\phi}$.

Define a map

$$
\Phi: \operatorname{Fix}(f) \longrightarrow \pi_{\phi}
$$

by

$$
x \longmapsto\left[\mu \cdot(f \circ \mu)^{-1} \cdot \tau^{-1}\right],
$$


where $x \in \operatorname{Fix}(f)$ and $\mu$ is a path in $M$ from $*$ to $x$. This map is well-defined and induces an injection

$$
\Phi: \operatorname{Fix}(f) / \sim \longrightarrow \pi_{\phi}
$$

It follows that $\operatorname{Fix}(f) / \sim$ is compact and discrete, and hence finite. Denote the fixed point classes by $F_{1}, \ldots, F_{s}$.

Next, assume that the fixed point set of $f$ is finite. Let $x$ be a fixed point. Let $U$ be an open neighborhood of $x$ in $M$ and $h: U \rightarrow \mathbb{R}^{n}$ a chart. Let $V$ be an open $n$-ball neighborhood of $x$ in $U$ such that $f(V) \subset U$. Then the fixed point index of $f$ at $x, i(f, x)$, is the degree of the map of pairs

$$
\left(\mathrm{id}-h f h^{-1}\right):(h(V), h(V)-\{h(x)\}) \rightarrow\left(\mathbb{R}^{n}, \mathbb{R}^{n}-\{0\}\right) .
$$

For a fixed point class $F_{k}$, define

$$
i\left(f, F_{k}\right)=\sum_{x \in F_{k}} i(f, x) \in \mathbb{Z} .
$$

Definition 2.1. The classical geometric Lefschetz invariant of $f$ with respect to the base point $*$ and the base path $\tau$ is

$$
L^{\mathrm{geo}}(f, *, \tau)=\sum_{k=1}^{s} i\left(f, F_{k}\right) \Phi\left(F_{k}\right) \in \mathbb{Z} \pi_{\phi},
$$

where $\mathbb{Z} \pi_{\phi}$ is the free abelian group generated by the set $\pi_{\phi}$.

2.2. The algebraic invariant. To construct the classical algebraic Lefschetz invariant, let $M$ be a finite connected CW complex and $f: M \rightarrow M$ a cellular map. Again, choose a base point $* \in M$ (a vertex of $M$ ) and a base path $\tau$ from $*$ to $f(*)$. Also, choose an orientation on each cell in $M$.

Let $p: \widetilde{M} \rightarrow M$ be the universal cover of $M$. The CW structure on $M$ lifts to a CW structure on $\widetilde{M}$. Choose a lift of the base point $*$ to a base point $\tilde{*} \in \widetilde{M}$, and lift the base path $\tau$ to a path $\tilde{\tau}$ such that $\tilde{\tau}(0)=\tilde{*}$. Then $f$ lifts to a cellular map $\tilde{f}: \widetilde{M} \rightarrow \widetilde{M}$ such that $\tilde{f}(\tilde{*})=\tilde{\tau}(1)$.

The group $\pi=\pi_{1}(M, *)$ acts on $\widetilde{M}$ on the left by covering transformations. For each cell $\sigma$ in $M$, choose a lift $\tilde{\sigma}$ in $\widetilde{M}$ and orient it compatibly with $\sigma$. Take the cellular chain complex $C(\widetilde{M})$ of $\widetilde{M}$. The action of $\pi$ on $\widetilde{M}$ makes $C_{k}(\widetilde{M})$ into a finitely generated free left $\mathbb{Z} \pi$-module with basis given by the chosen lifts of the oriented $k$-cells of $M$.

As in the geometric construction, $f$ and $\tau$ induce a homomorphism $\phi: \pi \rightarrow \pi$. Since $\tilde{f}$ is cellular, it induces a chain map $\tilde{f}_{k}: C_{k}(\widetilde{M}) \rightarrow C_{k}(\widetilde{M})$ which is $\phi$-linear, namely if $\tilde{\sigma}$ is a $k$-cell of $\widetilde{M}$ and $g \in \pi$ then $\tilde{f}_{k}(g \tilde{\sigma})=\phi(g) \tilde{f}_{k}(\tilde{\sigma})$. Classically, one represents $\tilde{f}_{k}$ by a matrix over $\mathbb{Z} \pi$ whose $(i, j)$ entry is the coefficient of $\tilde{\sigma}_{j}$ in the chain $\tilde{f}_{k}\left(\tilde{\sigma}_{i}\right)$, where $\tilde{\sigma}_{i}$ and $\tilde{\sigma}_{j}$ are $k$-cells. For each $k$, one can now take the trace of $\tilde{f}_{k}$, that is, the sum of the diagonal entries of the matrix which represents $\tilde{f}_{k}$. 
Definition 2.2. The classical algebraic Lefschetz invariant of $f$ with respect to the base point $*$ and the base path $\tau$ is

$$
L^{\mathrm{alg}}(f, *, \tau)=\sum_{k \geq 0}(-1)^{k} q\left(\operatorname{trace}\left(\tilde{f}_{k}\right)\right) \in \mathbb{Z} \pi_{\phi},
$$

where $q: \mathbb{Z} \pi \rightarrow \mathbb{Z} \pi_{\phi}$ is the map sending $g \in \pi$ to its semiconjugacy class.

2.3. Hattori-Stallings trace. In the classical algebraic construction of the Lefschetz invariant above, Reidemeister viewed $\tilde{f}_{k}$ as a matrix and took its trace, the sum of the diagonal entries, to define $L^{\text {alg }}(f)$. In our generalizations, we will need to use a more sophisticated trace map, namely the Hattori-Stallings trace. Since on finitely generated free modules, the Hattori-Stallings trace agrees with the usual trace of a matrix, we could use it in the classical case as well. We introduce the classical Hattori-Stallings trace here. (For the special case when $M=R$, see $[1,2,9]$.)

Let $R$ be a ring, $M$ an $R$-bimodule, and $P$ a finitely generated projective left $R$-module. Let $P^{*}=\operatorname{Hom}_{R}(P, R)$ be the dual of $P$. Let $[R, M]$ denote the abelian subgroup of $M$ generated by elements of the form $r m-m r$, for $r \in R$ and $m \in M$. The Hattori-Stallings trace map, tr is given by the following composition:

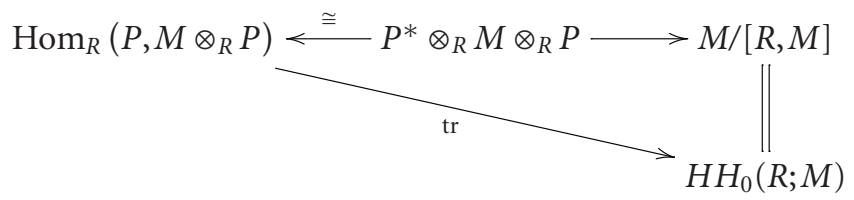

The map $P^{*} \otimes_{R} M \otimes_{R} P \rightarrow \operatorname{Hom}_{R}\left(P, M \otimes_{R} P\right)$ is given by $\alpha \otimes m \otimes p \mapsto\left(p_{1} \mapsto \alpha\left(p_{1}\right)(m \otimes\right.$ $p)$ ). The map $P^{*} \otimes_{R} M \otimes_{R} P \rightarrow M /[R, M]$ is given by $\alpha \otimes m \otimes p \mapsto \alpha(p) m$.

The fact that the first map is an isomorphism is an application of the following lemma.

Lemma 2.3. Let $R$ be a ring, $P$ a finitely generated projective right $R$-module, and $N a$ left $R$-module. Define $f_{P}: P^{*} \otimes_{R} N \rightarrow \operatorname{Hom}_{R}(P, N)$ by $f_{P}(\alpha, n)(p)=\alpha(p) n$. Then $f_{P}$ is an isomorphism of groups.

Proof. Note that $f_{R}: R^{*} \otimes_{R} N \rightarrow \operatorname{Hom}_{R}(R, N)$ is an isomorphism with inverse given by $(g$ : $R \rightarrow N) \mapsto \mathrm{id}_{R} \otimes_{R} g\left(1_{R}\right)$. The result follows from the fact that $f_{(-)}:(-)^{*} \otimes_{R} N \rightarrow \operatorname{Hom}_{R}(-$, $N)$ preserves finite direct sums.

\section{Background on groups and ringoids}

In this section, we generalize to the "oid" setting the basic algebraic definitions and results which we will need for our constructions. This treatment is based on [7, Section 9], though we have developed additional material as needed. In particular, in Section 3.2, we generalize the Hattori-Stallings trace.

We use the following notation. If $C$ is a category, denote the collection of objects in $C$ by $\mathrm{Ob}(C)$. If $x$ and $y$ are objects in $C$, denote the collection of maps from $x$ to $y$ in $C$ by $C(x, y)$. The category of sets will be denoted Sets, the category of abelian groups will be denoted $\mathbf{A b}$, and the category of left $R$-modules will be denoted $R$-mod.

Throughout, "ring" will mean an associative ring with unit. 


\subsection{General definitions and results}

3.1.1. Groupoids and ringoids. Let $G$ be a group. We may view $G$ as a category, denoted by $\mathbf{G}$, in which there is one object $*$, and for which all of the maps are isomorphisms. Each map corresponds to an element of $G$ with composition of maps corresponding to the multiplication in the group. This idea generalizes to define a groupoid.

Definition 3.1. A groupoid $G$ is a small category (the objects form a set) such that all maps are isomorphisms.

The analogous game can be played with rings in order to define a ringoid, also known as a linear category or as a small category enriched in the category of abelian groups.

Definition 3.2. A ringoid $\mathscr{R}$ is a small category such that for each pair of objects $x$ and $y$, $\mathscr{R}(x, y)$ is an abelian group and the composition function $\mathscr{R}(y, z) \times \mathscr{R}(x, y) \rightarrow \mathscr{R}(x, z)$ is bilinear.

Example 3.3. Recall that if $H$ is a group, then the group ring $\mathbb{Z} H$ is the free abelian group generated by $H$. This group ring construction can be generalized to a "groupoid ringoid" (though we will call it the group ring): let $G$ be a groupoid and $R$ a ring. The group ring of $G$ with respect to $R$, denoted $R G$, is the category with the same objects as $G$, but with maps given by $R G(x, y)=R(G(x, y))$, the free $R$-module generated by the set $G(x, y)$.

3.1.2. Modules. For the remainder of this paper, unless otherwise noted, let $G$ be a groupoid and let $R$ be a commutative ring. While much of the following can be done in terms of a ringoid $\mathscr{R}$, we will restrict our attention to group rings $R G$.

Definition 3.4. A left $R G$-module is a (covariant) functor $M: G \rightarrow R$-mod. A right $R G$ modules is a (covariant) functors $G^{\text {op }} \rightarrow R$-mod.

Definition 3.5. Let $M$ and $N$ be $R G$-modules. An $R G$-module homomorphism from $M$ to $N$ is a natural transformation from $M$ to $N$. The set of all $R G$-module homomorphisms from $M$ to $N$ is denoted by $\operatorname{Hom}_{R G}(M, N)$.

Let $R G$-mod denote the category of left $R G$-modules, and let mod- $R G$ denote the category of right $R G$-modules.

Definition 3.6. Let $M$ and $N$ be $R G$-modules. The direct sum $M \oplus N$ of $M$ and $N$ is the left $R G$-module defined on an object $x$ by $(M \oplus N)(x)=M(x) \oplus N(x)$ and on a map $g: x \rightarrow y$ by $(M \oplus N)(g)=M(g) \oplus N(g)$.

Definition 3.7. Let $N$ be a left $R G$-module and $M$ a right $R G$-module. Define the tensor product over $R G$ of $M$ and $N$ to be the abelian group

$$
M \otimes_{R G} N=P / Q
$$

where $P$ is the abelian group

$$
P=\bigoplus_{x \in \operatorname{Ob}(G)} M(x) \otimes_{R} N(x)
$$


6 A base-point-free definition of the Lefschetz invariant

and $Q$ is the subgroup of $P$ generated by

$$
\{M(f)(m) \otimes n-m \otimes N(f)(n) \mid m \in M(y), n \in N(x), f \in R G(x, y)\} .
$$

Proposition 3.8. Let $M, N$, and $P$ be RG-modules. Then

$$
\operatorname{Hom}_{R G}(M \oplus N, P) \cong \operatorname{Hom}_{R G}(M, P) \oplus \operatorname{Hom}_{R G}(N, P) .
$$

Proposition 3.9. Let $M, N$, and $P$ be RG-modules. Then

$$
(M \oplus N) \otimes_{R G} P \cong\left(M \otimes_{R G} P\right) \oplus\left(N \otimes_{R G} P\right) .
$$

Definition 3.10. Given an $R G$-bimodule $M$, define $M /[R G, M]$ to be the $R$-module

$$
\left(\bigoplus_{x \in \mathrm{Ob}(G)} M(x, x)\right) /\left\{m-M\left(g, g^{-1}\right)(m) \mid g: x \longrightarrow y, m \in M(x, x)\right\} .
$$

Call this the zero dimensional Hochschild homology of $R G$ with coefficients in $M$, denoted by

$$
H H_{0}(R G ; M)
$$

Next, we define free $R G$-modules. First, we need the following notions.

Given a category $C$, we can view $\mathrm{Ob}(C)$ as the subcategory of $C$ whose objects are the same as the objects of $C$, but whose maps are only the identity maps. A covariant (contravariant) functor $\mathrm{Ob}(C) \rightarrow$ Sets will be called a left (right) $\mathrm{Ob}(C)$-set. A map of $\mathrm{Ob}(C)$ sets is a natural transformation. Let $\mathrm{Ob}(C)$-Sets denote the category of left $\mathrm{Ob}(C)$-sets, and let Sets- $\mathrm{Ob}(C)$ denote the category of right $\mathrm{Ob}(C)$-sets.

Given either a left or right $\mathrm{Ob}(C)$-set $B$, let

$$
\mathscr{B}=\bigsqcup_{x \in \mathrm{Ob}(C)} B(x)
$$

where $\sqcup$ denotes disjoint union, and let

$$
\beta: \mathscr{P} \longrightarrow \mathrm{Ob}(C)
$$

send $b$ to $x$ if $b \in B(x)$. Given $\mathrm{Ob}(C)$-sets $B$ and $B^{\prime}$, we say $B$ is an $\mathrm{Ob}(C)$-subset of $B^{\prime}$ if for every $x \in \mathrm{Ob}(C), B(x) \subset B^{\prime}(x)$.

Suppose $C$ is a small category and $D$ is a category equipped with a "forgetful functor" $D \rightarrow$ Sets. For a functor $F: C \rightarrow D$, let $|F|: \operatorname{Ob}(C) \rightarrow$ Sets be the composition $\operatorname{Ob}(C) \hookrightarrow$ $C \rightarrow D \rightarrow$ Sets, where the functor $D \rightarrow$ Sets is the forgetful functor. In particular, $|-|$ : $R G$-mod $\rightarrow \mathrm{Ob}(C)$-Sets and $|-|: \bmod -R G \rightarrow \operatorname{Sets}-\mathrm{Ob}(G)$.

Definition 3.11. For each $x \in \mathrm{Ob}(G)$, define a left $R G$-module $\overline{R G}_{x}=R G(x,-)$ by $\overline{R G}_{x}(y)=R G(x, y)$. For a map $g: y \rightarrow z$ in $G$, let $\overline{R G}_{x}(g)=g \circ(-)$. Define a right $R G$ module $\overline{R G}^{x}=R G(-, x)$ similarly. 
Definition 3.12. Define a functor $\overline{R G}_{(-)}: \mathrm{Ob}(G)$-Sets $\rightarrow R G$-mod by

$$
\overline{R G}_{B}=\bigoplus_{b \in \mathscr{B}} \overline{R G}_{\beta(b)}=\bigoplus_{b \in \mathscr{B}} R G(\beta(b),-) .
$$

Similarly, define $\overline{R G}^{(-)}$: Sets- $\mathrm{Ob}(G) \rightarrow \bmod -R G$ by

$$
\overline{R G}^{B}=\bigoplus_{b \in \mathscr{P}} \overline{R G}^{\beta(b)}=\bigoplus_{b \in \mathscr{P}} R G(-, \beta(b)) .
$$

Proposition 3.13. The functor $\overline{R G}_{(-)}$is a left adjoint to the functor $|-|: R G$-mod $\rightarrow$ $\mathrm{Ob}(G)$-Sets. The functor $\overline{R G}^{(-)}$is a left adjoint to $|-|: \bmod -R G \rightarrow \operatorname{Sets}-\mathrm{Ob}(G)$.

Proof. For an $\mathrm{Ob}(G)$-set $B$ and a left $R G$-module $M$, define a set map $\psi=\psi_{B, M}$ : $R G-\bmod \left(\overline{R G}_{B}, M\right) \rightarrow \mathrm{Ob}(G)-\operatorname{Sets}(B,|M|)$ by $\psi(\eta)_{y}(b)=\eta_{y}\left(\mathrm{id}_{y}\right) \in|M(y)|$, where $\eta:$ $\overline{R G}_{B} \rightarrow M$ is a natural transformation and $b \in B(y)$. Then $\psi$ is a bijection whose inverse is defined in the most obvious way.

Notice that for each $\mathrm{Ob}(G)$-set $B$, we get a natural transformation $\eta_{B}=\psi\left(\operatorname{id}_{\overline{R G}_{B}}\right): B \rightarrow$ $\left|\overline{R G}_{B}\right|$ which is universal. This leads to the following definition of a free $R G$-module with base $B$.

Definition 3.14. An $R G$-module $M$ is free with base an $\mathrm{Ob}(G)$-set $B \subset|M|$ if for each $R G$-module $N$ and natural transformation $f: B \rightarrow|N|$ there is a unique natural transformation $F: M \rightarrow N$ with $|F| \circ i=f$, where $i$ is the inclusion $B \rightarrow|M|$.

Example 3.15. The $R G$-module $\overline{R G}_{x}$ is a free left $R G$-module with base $B_{x}: \mathrm{Ob}(G) \rightarrow$ Sets given by

$$
B_{x}(y)= \begin{cases}\{x\} & \text { if } y=x \\ \varnothing & \text { if } y \neq x\end{cases}
$$

If $B$ is any $\mathrm{Ob}(G)$-set, $\overline{R G}_{B}=\bigoplus_{b \in \mathscr{P}} \overline{R G}_{\beta(b)}=\bigoplus_{b \in \mathscr{P}} R G(\beta(b),-)$ is a free $R G$-module with base $B$.

Let $M$ be an $R G$-module. Let $S$ be an $\operatorname{Ob}(G)$-subset of $|M|$ and let $\operatorname{Span}(S)$ be the smallest $R G$-submodule of $M$ containing $S$,

$$
\operatorname{Span}(S)=\cap\{N \mid N \text { is an } R G \text {-submodule of } M, S \subset N\} .
$$

Definition 3.16. Say that $M$ is generated by $S$ if $M=\operatorname{Span}(S)$, and $M$ is finitely generated if $S$ is finite.

Proposition 3.17. If $M$ is a left $R G$-module, and $B$ is an $\mathrm{Ob}(G)$-subset of $|M|$, then $\operatorname{Span}(B)$ is the image of the unique natural transformation $\tau: \overline{R G}_{B} \rightarrow$ extending id $: B \rightarrow$ $B \subset|M|$. Furthermore, $M$ is generated by $B$ if $\tau$ is surjective.

Proposition 3.18. Let $B$ be an $\mathrm{Ob}(G)$-set. If $M$ is a free left $R G$-module with base $B$, then $M$ is generated by $B$. In particular, there is a natural equivalence $\tau: \overline{R G}_{B} \rightarrow M$. 
8 A base-point-free definition of the Lefschetz invariant

Proof. Define $\tau: \overline{R G}_{B} \rightarrow M$. For $x \in \mathrm{Ob}(G)$, let

$$
\tau_{x}: \overline{R G}_{B}(x)=\bigoplus_{b \in \mathscr{B}} R G(\beta(b), x) \longrightarrow M(x)
$$

be given by $(g: \beta(b) \rightarrow x) \mapsto M(g)(b)$. To construct an inverse natural transformation, define $\eta: B \rightarrow\left|\overline{R G}_{B}\right|$ by setting $\eta_{x}(b)=\mathrm{id}_{x}$. Since $M$ is free with base $B, \eta$ extends to a unique natural transformation $M \rightarrow \overline{R G}_{B}$.

Definition 3.19. An $R G$-module $P$ is projective if it is the direct summand of a free $R G$ module.

\subsubsection{Bimodules.}

Definition 3.20. An RG-bimodule is a (covariant) functor

$$
M: G \times G^{\mathrm{op}} \longrightarrow R \text {-mod }
$$

Denote the category of $R G$-bimodules by $R G$-bimod.

Example 3.21. Let $\overline{R G}$ be $R G$ with the following $R G$-bimodule structure. For $(x, y) \in$ $G \times G^{\text {op }}$, set $\overline{R G}(x, y)=R G(y, x)$. Notice the change in the order of $x$ and $y$. For maps $g: x \rightarrow x^{\prime}$ in $G$ and $h: y \rightarrow y^{\prime}$ in $G^{\text {op }}$, set $\overline{R G}(g, h)=g \circ(-) \circ h: R G(y, x) \rightarrow R G\left(y^{\prime}, x^{\prime}\right)$.

We would like to be able to view an $R G$-bimodule $N$ as either a right or a left $R G$ module. However, there is no canonical way to do so as each choice of object in $G$ produces a different left and a right $R G$-module structure on $N$. Instead, we define two functors: (-) ad and ad(-). In essence, $N$ ad encapsulates all of the right $R G$-module structures on $N$ induced by objects of $G$, and ad $N$ encapsulates all of the left $R G$-module structure on $N$.

Definition 3.22. Define a covariant functor

$$
(-) \text { ad }: R G \text {-bimod } \longrightarrow(\bmod -R G)^{G}
$$

as follows. Let $N$ be an $R G$-bimodule. For $x \in \mathrm{Ob}(G)$, let

$$
N \operatorname{ad}(x)=N(x,-)
$$

For $g$ a map in $G$, let

$$
N \operatorname{ad}(g)=N(g,-)
$$

Explicitly, $N \operatorname{ad}(x): G^{\text {op }} \rightarrow R$-mod is given by $N \operatorname{ad}(x)(y)=N(x, y)$ and $N \operatorname{ad}(x)(h)=$ $N\left(\mathrm{id}_{x}, h\right)$ for $h: y \rightarrow z$ a map in $G^{\mathrm{op}}$.

Definition 3.23. Define a covariant functor

$$
\operatorname{ad}(-): R G \text {-bimod } \longrightarrow(R G-\bmod )^{G^{\mathrm{op}}}
$$


as follows. Let $N$ be an $R G$-bimodule. For $x \in \mathrm{Ob}\left(G^{\mathrm{op}}\right)$, let

$$
\operatorname{ad} N(x)=N(-, x)
$$

For $g$ a map in $G^{\text {op }}$, let

$$
\operatorname{ad} N(g)=N(-, g)
$$

Explicitly, $\operatorname{ad} N(x): G \rightarrow R$-mod is given by $\operatorname{ad} N(x)(y)=N(y, x)$ and $\operatorname{ad} N(x)(h)=N(h$, $\mathrm{id}_{x}$ ) for $h: y \rightarrow z$ a map in $G$.

Example 3.24. Apply the ad functors to the $R G$-bimodule $\overline{R G}$. For instance, if $x \in \mathrm{Ob}(G)$, then $\operatorname{ad} \overline{R G}(x)=R G(x,-)=\overline{R G}_{x}$. Hence, ad $\overline{R G}(x): G \rightarrow R$-mod, with $\operatorname{ad} \overline{R G}(x)(y)=$ $R G(x, y)$ and $\operatorname{ad} \overline{R G}(x)(h)=h \circ(-)$ for $h: y \rightarrow z$ a map in $G$. Also, for $g: x \rightarrow x^{\prime}$ a map in $G^{\mathrm{op}}$, ad $\overline{R G}(g)=\overline{R G}(-, g): R G(x,-) \rightarrow R G\left(x^{\prime},-\right)$ is the natural transformation of left $R G$-modules given by ad $\overline{R G}(g)_{y}=(-) \circ g: R G(x, y) \rightarrow R G\left(x^{\prime}, y\right)$.

Next, if $N$ is an $R G$-bimodule and $M$ is an $R G$-module, we define $\operatorname{Hom}_{R G}(N, M)$, $\operatorname{Hom}_{R G}(M, N), N \otimes_{R G} M_{l}$ and $M_{r} \otimes_{R G} N$ in such a way that they are also $R G$-modules, as one would expect. Let $M_{l}$ (resp., $M_{r}$ ) denote a left (resp., right) $R G$-module.

Definition 3.25. Let $N$ be an $R G$-bimodule. $\operatorname{Hom}_{R G}\left(M_{l}, N\right)$ is defined to be the right $R G$ module given by the composition

$$
G^{\mathrm{op}} \stackrel{\operatorname{adN}}{\longrightarrow} R G-\bmod \stackrel{\operatorname{Hom}_{R G}\left(M_{l},-\right)}{\longrightarrow} R-\bmod .
$$

$\operatorname{Hom}_{R G}\left(N, M_{l}\right)$ is defined to be the left $R G$-module given by the composition

$$
G^{\text {op }} \stackrel{\text { adN }}{\longrightarrow} R G-\bmod \stackrel{\operatorname{Hom}_{R G}\left(-, M_{l}\right)}{\longrightarrow} R-\bmod
$$

$\operatorname{Hom}_{R G}\left(M_{r}, N\right)$ is defined to be the left $R G$-module given by the composition

$$
G \stackrel{N \text { ad }}{\longrightarrow} \bmod -R G \stackrel{\operatorname{Hom}_{R G}\left(M_{r},-\right)}{\longrightarrow} R-\bmod
$$

$\operatorname{Hom}_{R G}\left(N, M_{r}\right)$ is defined to be the right $R G$-module given by the composition

$$
G \stackrel{N \text { ad }}{\longrightarrow} \bmod -R G \stackrel{\operatorname{Hom}_{R G}\left(-, M_{r}\right)}{\longrightarrow} R-\bmod
$$

Definition 3.26. Let $N$ be an $R G$-bimodule. Define $N \otimes_{R G} M_{l}$ to be the left $R G$-module given by the composition

$$
G \stackrel{N \text { ad }}{\longrightarrow} \bmod -R G \stackrel{(-) \otimes_{R G} M_{l}}{\longrightarrow} R \text {-mod. }
$$

Define $M_{r} \otimes_{R G} N$ to be the right $R G$-module given by the composition

$$
G^{\text {op }} \stackrel{\text { adN }}{\longrightarrow} R G-\bmod \stackrel{M_{r} \otimes_{R G}(-)}{\longrightarrow} R-\bmod .
$$


Applying the above definitions to the $R G$-bimodule $\overline{R G}$, we get the results for Hom and tensor product which we would expect from algebra. These next three propositions justify viewing $\overline{R G}$ as "the free rank-one" $R G$-module. Notice that it is not, however, a free $R G$-module. The proofs are straightforward and left to the reader.

Proposition 3.27. Given an $R G$-module $M, \operatorname{Hom}_{R G}(\overline{R G}, M) \cong M$ as RG-modules.

Proposition 3.28. Given a left $R G$-module $M, \overline{R G} \otimes_{R G} M \cong M$ as left $R G$-modules.

Proposition 3.29. Given right $R G$-module $M, M \otimes_{R G} \overline{R G} \cong M$ as right $R G$-modules.

In particular, we can now define the dual of an $R G$-module.

Definition 3.30. Let $M$ be a left (right) $R G$-module. The dual of $M$ is the right (left) $R G$ module $M^{*}=\operatorname{Hom}_{R G}(M, \overline{R G})$.

Proposition 3.31. Let $M$ and $N$ be RG-modules. Then there is a natural equivalence $(M \oplus$ $N)^{*} \cong M^{*} \oplus N^{*}$.

\subsubsection{Chain complexes.}

Definition 3.32. An $R G$-chain complex is a (covariant) functor $C .: G \rightarrow \mathrm{Ch}(R)$, where $\mathrm{Ch}(R)$ is the category of chain complexes over the ring $R$.

LEMma 3.33. The following are equivalent:

(i) $C$. is an $R G$-chain complex;

(ii) there exist a family $\left\{C_{n}\right\}$ of $R G$-modules together with a family of natural transformations $\left\{d_{n}: C_{n} \rightarrow C_{n-1}\right\}$, called differentials, such that $d_{n-1} \circ d_{n}=0$.

Using the second characterization of $R G$-chain complexes, we can now define finitely generated projective chain complexes, chain maps and chain homotopies in the usual manner.

Definition 3.34. An $R G$-chain complex $P$. is said to be a finitely generated projective if each $P_{n}$ is a finitely generated projective $R G$-module and $P$. is bounded (i.e., $P_{n}=0$ for all but a finite number of $n)$. Let $\mathscr{P}(R G)$ denote the subcategory of finitely generated projective $R G$-chain complexes.

Definition 3.35. An $R G$-chain map $f: C$. $\rightarrow D$. is a family $\left\{f_{n}: C_{n} \rightarrow D_{n}\right\}$ of natural transformations such that $d_{n}^{\prime} \circ f_{n}=f_{n-1} \circ d_{n}$ for all $n$, where the $d_{n}$ are the differentials of $C$. and the $d_{n}^{\prime}$ are the differentials of $D$.

Definition 3.36. Two RG-chain maps $f: C . \rightarrow D$. and $g: C . \rightarrow D$. are $R G$-chain homotopic, denoted by $f \sim_{\text {ch }} g$, if there exists a family $\left\{s_{n}: C_{n} \rightarrow D_{n-1}\right\}$ of natural transformations such that

$$
f_{n}-g_{n}=d_{n+1}^{\prime} \circ s_{n}+s_{n-1} \circ d_{n}
$$

Definition 3.37. Two $R G$-chain complexes $C$. and $D$. are chain homotopy equivalent if there exist $R G$-chain maps $f: C$. $\rightarrow D$. and $g: D$. $\rightarrow C$. such that $f \circ g \sim_{\text {ch }} \operatorname{id}_{D}$. and $g \circ$ $f \sim_{c h} \operatorname{id}_{C}$. In this case, $f$ is said to be a chain homotopy equivalence. 
3.1.5. Everything $\alpha$-twisted. For the remainder of the paper, let $\alpha: G \rightarrow G$ be a functor. We can use $\alpha$ to create an " $\alpha$-twisted" version of many of our algebraic objects.

Definition 3.38. Define an $R G$-bimodule ${ }_{\alpha} R G: G \times G^{\text {op }} \rightarrow R$-mod by

$$
{ }_{\alpha} R G(x, y)=R G(y, \alpha(x))
$$

for $x, y \in \mathrm{Ob}(G)$, and

$$
{ }_{\alpha} R G(g, h)=\alpha(g) \circ(-) \circ h
$$

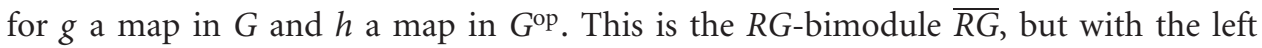
module structure twisted by $\alpha$.

Definition 3.39. Let $M$ and $N$ be RG-modules. An $\alpha$-linear homomorphism $M \rightarrow N$ is defined to be a natural transformation $\eta: M \rightarrow N \circ \alpha$. A chain map $f: C . \rightarrow D$. of $R G$ chain complexes is called $\alpha$-linear if for each $n, f_{n}$ is $\alpha$-linear.

LEMma 3.40. Given left RG-modules $P$ and $Q$, there is an isomorphism

$$
\operatorname{Hom}_{R G}(P, Q \circ \alpha) \cong \operatorname{Hom}_{R G}\left(P,{ }_{\alpha} R G \otimes_{R G} Q\right) .
$$

Definition 3.41. Let $M$ be an $R G$-module. The $\alpha$-dual of $M$ is

$$
M^{\alpha}=\operatorname{Hom}_{R G}\left(M,{ }_{\alpha} R G\right) .
$$

Proposition 3.42. Let $P$ and $Q$ be RG-modules and $N$ an RG-bimodule. Then there is a natural equivalence of $R G$-modules

$$
\operatorname{Hom}_{R G}(P \oplus Q, N) \cong \operatorname{Hom}_{R G}(P, N) \oplus \operatorname{Hom}_{R G}(Q, N) .
$$

Corollary 3.43. Let $P$ and $Q$ be left $R G$-modules. Then there is a natural equivalence

$$
(P \oplus Q)^{\alpha} \cong P^{\alpha} \oplus Q^{\alpha} .
$$

3.2. Generalized Hattori-Stallings trace. In this section, we define an $\alpha$-twisted HattoriStallings trace for $R G$-modules. One can define a more general Hattori-Stallings trace for $R G$-modules, in the same manner as the classical definition given in Section 2.3. However, as we will not need this more general form, we will concern ourselves only with the special $\alpha$-twisted case. We also extend the trace to $R G$-chain complexes.

3.2.1. Definition and commutativity. Given left $R G$-modules $N$ and $P$, define an $R$-module homomorphism

$$
\phi_{P}=\phi_{P, N}: P^{\alpha} \otimes_{R G} N \longrightarrow \operatorname{Hom}_{R G}(P, N \circ \alpha)
$$

by letting: $\phi_{P}(\tau \otimes n): P \rightarrow N \circ \alpha$ be the natural transformation given by

$$
\phi_{P}(\tau \otimes n)_{y}(p)=N\left(\tau_{y}(p)\right)(n)
$$

where $\tau \in P^{\alpha}(x), m \in N(x), p \in P(y)$, and $x, y \in \mathrm{Ob}(G)$. 
PROPOSITION 3.44. If $P$ is a finitely generated projective $R G$-module, then $\phi_{P}$ is an isomorphism.

The proof will use the following three lemmas.

Lemma 3.45. Given $x \in \mathrm{Ob}(G)$, then $\phi_{\overline{R G}_{x}}$ is an isomorphism.

Proof. Write $\phi$ for $\phi_{\overline{R G}_{x}}$. Define

$$
\psi: \operatorname{Hom}_{R G}\left(\overline{R G}_{x}, N \circ \alpha\right) \longrightarrow \overline{R G}_{x}^{\alpha} \otimes_{R G} N
$$

by

$$
\eta \longmapsto \bar{\alpha} \otimes \eta_{x}\left(\mathrm{id}_{x}\right)
$$

where $\eta: \overline{R G}_{x} \rightarrow N \circ \alpha$ is a natural transformation. Here, $\bar{\alpha} \in P^{\alpha}(x)$ is the natural transformation induced by $\alpha$, that is, $\bar{\alpha}_{y}(f)=\alpha(f)$ for $y \in \mathrm{Ob}(G)$ and $f \in R G(x, y)$.

It is easy to show that $\phi \circ \psi=\mathrm{id}$ and $\psi \circ \phi=\mathrm{id}$.

LemmA 3.46. If $P$ and $Q$ are left $R G$-modules, then $\phi_{P \oplus Q}=\phi_{P} \oplus \phi_{Q}$.

Proof. Consider the following diagram:

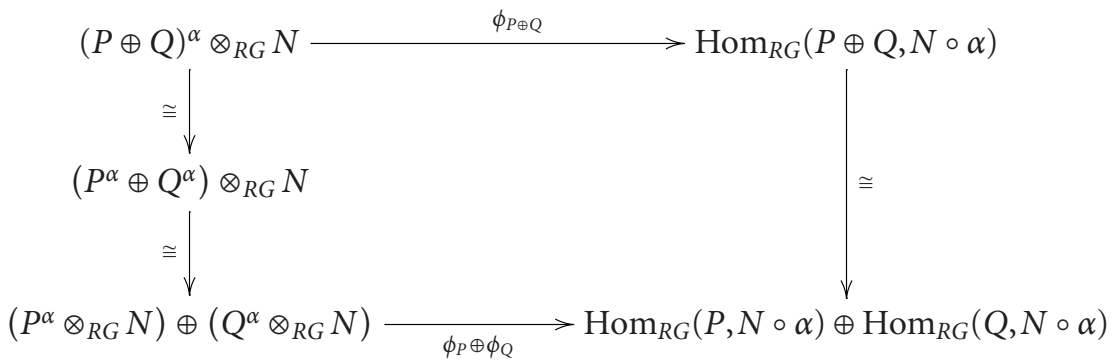

The vertical isomorphisms are as in Propositions 3.8 and 3.9 and Corollary 3.43. Using those isomorphism, one can see that the diagram commutes.

Lemma 3.47. Let $P$ and $Q$ be left $R G$-modules and let $N=P \oplus Q$. If $\phi_{N}$ is an isomorphism, then $\phi_{P}$ is an isomorphism also.

Proof. By the previous lemma, $\phi_{N}=\phi_{P} \oplus \phi_{Q}$. The result follows immediately.

Proof of Proposition 3.44. The proof is in two steps.

Step 1. Suppose that $P$ is a finitely generated free $R G$-module. Then $P$ is naturally equivalent to $\overline{R G}_{B}=\bigoplus_{b \in \mathscr{B}} \overline{R G}_{\beta(b)}$ for some $\mathrm{Ob}(G)$-set $B$. By Lemma 3.46, $\phi_{P}=\bigoplus_{b \in \mathscr{B}} \phi_{\overline{R G}_{\beta(b)}}$, and by Lemma 3.45, it is an isomorphism.

Step 2. Suppose that $P$ is a finitely generated projective $R G$-modules and so $P$ is a direct summand of a finitely generated free $R G$-module. Combining Step 1 and Lemma 3.47 we see that $\phi_{P}$ is an isomorphism. 
For $P$ a left $R G$-module, define an $R$-module homomorphism

$$
P^{\alpha} \otimes_{R G} P \longrightarrow{ }_{\alpha} R G /\left[R G,{ }_{\alpha} R G\right]
$$

by $\tau \otimes p \mapsto \tau_{x}(p)$ where $\tau \in P^{\alpha}(x)$ and $p \in P(x)$.

Definition 3.48. Let $P$ be a finitely generated projective left $R G$-module. The HattoriStallings trace, denoted by tr, is the composition

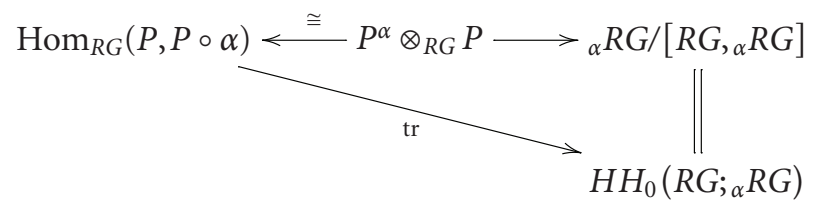

where the isomorphism is the map $\phi_{P}$ and the unadorned arrow is the homomorphism described above.

Proposition 3.49 (commutativity). Let $P$ and $Q$ be finitely generated projective left $R G$ modules. If $f \in \operatorname{Hom}_{R G}(P, Q \circ \alpha)$ and $g \in \operatorname{Hom}_{R G}(Q, P)$, then

$$
\operatorname{tr}(f \circ g)=\operatorname{tr}(g \circ \alpha \circ f) .
$$

Proof. The result follows from commutativity of three diagrams.

The first diagram is

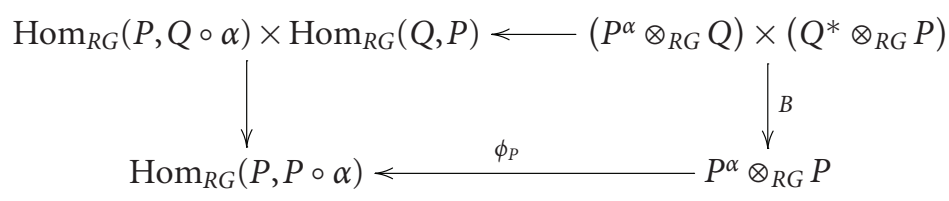

where $B$ is given by $(\eta \otimes p) \times(\tau \otimes q) \mapsto(\alpha \circ \eta) \otimes Q\left(\tau_{y}(p)\right)(q)$, the unlabelled vertical map is given by $(f, g) \mapsto g \circ \alpha \circ f$ and the unlabelled horizontal map is $\phi_{P^{\alpha}, Q} \times \phi_{Q, P}$.

The second diagram is gotten by transposing the products in the first diagram.

The third diagram is

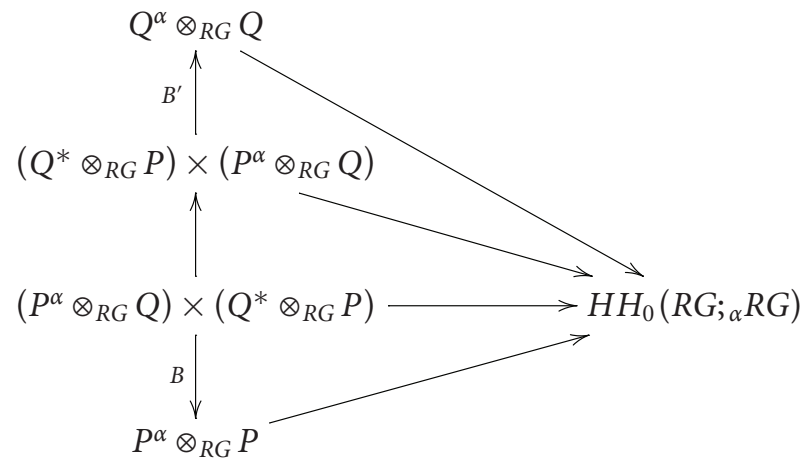


where the unlabelled arrow is transposition, $B^{\prime}$ is analogous to $B$, and the other maps are defined in the obvious ways.

3.2.2. For connected groupoids. Consider the following setup. Let $G$ be a connected groupoid, that is, one for which there exists a map between any two objects. Let $\alpha: G \rightarrow G$ be a functor and let $P$ be a finitely generated projective left $R G$-module. Choose an object $*$ of $G$ and choose a map $\tau: * \rightarrow \alpha(*)$ in $G$.

Let $R G(*)$ be the subcategory of $R G$ with a single object, $*$, and with maps given by the maps in $R G$ from $*$ to $*$. Then the inclusion $R G(*) \rightarrow R G$ is an equivalence of categories. The proof amounts to choosing a map $\mu_{x}: * \rightarrow x$ for each $x \in \operatorname{Ob}(G)$. For each $x$, we fix a choice of $\mu_{x}$.

The functor $\alpha$ induces a functor $\alpha_{\tau}: R G(*) \rightarrow R G(*)$ which maps the object $*$ to itself. If $g: * \rightarrow *$, let $\alpha_{\tau}(g)=\tau^{-1} \circ \alpha(g) \circ \tau$. In the obvious way, the $R G$-module $P$ induces a finitely generated projective left $R G(*)$-module, denoted $P(*)$. A natural transformation $\beta \in \operatorname{Hom}_{R G}(P, P \circ \alpha)$ induces a natural transformation $\beta_{\tau}=P\left(\tau^{-1}\right) \circ \beta_{*} \in$ $\operatorname{Hom}_{R G(*)}\left(P(*), P(*) \circ \alpha_{\tau}\right)$.

Lemma 3.50. There is an isomorphism of groups

$$
A: H H_{0}\left(R G(*) ;{ }_{\alpha_{\tau}} R G(*)\right) \longrightarrow H H_{0}\left(R G ;{ }_{\alpha} R G\right)
$$

given by $A(m)=\tau \circ m$ for $m \in H H_{0}\left(R G(*) ; \alpha_{\tau} R G(*)\right)$.

Proposition 3.51. The Hattori-Stallings trace of $\beta_{\tau}$ and $\beta$ are equivalent, that is,

$$
A\left(\operatorname{tr}\left(\beta_{\tau}\right)\right)=\operatorname{tr}(\beta)
$$

Proof. Given $\eta \in P^{\alpha}(x)$ for some $x \in \mathrm{Ob}(G)$, define $\bar{\eta}: P(*) \rightarrow R G(*, *) \in P(*)^{\alpha_{\tau}}$ by $\bar{\eta}(p)=\tau^{-1} \circ \eta_{*}(p) \circ \mu_{x}$, where $p \in P(*)$. This gives us a map $P^{\alpha} \rightarrow P(*)^{\alpha_{\tau}}$.

Define a map $B: P^{\alpha} \otimes_{R G} P \rightarrow P(*)^{\alpha_{\tau}} \otimes R G(*) P(*)$ by $\eta \otimes p \mapsto \bar{\eta} \otimes P\left(\mu_{x}^{-1}\right)(p)$, where $\eta \in P^{\alpha}(x)$ and $p \in P(x)$ for some $x \in \mathrm{Ob}(G)$. Define a map $C: \operatorname{Hom}_{R G}(P, P \circ \alpha) \rightarrow$ $\operatorname{Hom}_{R G(*)}\left(P(*), P(*) \circ \alpha_{\tau}\right)$ by $\gamma \mapsto \gamma_{\tau}=P\left(\tau^{-1}\right) \circ \gamma_{*}$ for $\gamma \in \operatorname{Hom}_{R G}(P, P \circ \alpha)$.

Commutativity of the following two diagrams implies that $A\left(\operatorname{tr}\left(\beta_{\tau}\right)\right)=\operatorname{tr}(\beta)$.

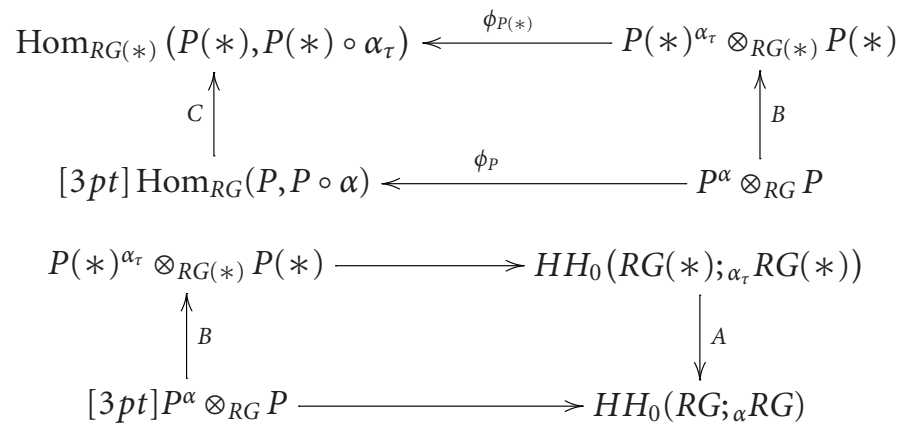

Notice that $A\left(\operatorname{tr}\left(\beta_{\tau}\right)\right)$ is independent of the choices of maps $\mu_{x}$. 
3.2.3. For chain complexes. We begin with the general case.

Definition 3.52. Let $P$. be a finitely generated projective $R G$-chain complex. Define the Hattori-Stallings trace

$$
\operatorname{Tr}: \operatorname{Hom}_{\mathscr{P}(R G)}\left(P_{.}, P . \circ \alpha\right) \longrightarrow H H_{0}\left(R G ;{ }_{\alpha} R G\right)
$$

by

$$
f \longmapsto \sum_{i}(-1)^{i} \operatorname{tr}\left(f_{i}\right)
$$

where $f: P . \rightarrow P . \circ \alpha$ is given by the family $\left\{f_{i} \in \operatorname{Hom}_{R G}\left(P_{i}, P_{i} \circ \alpha\right)\right\}$.

Commutativity follows from commutativity of the Hattori-Stallings trace for $R G$ modules.

Proposition 3.53 (commutativity). Let $P$. and Q. be finitely generated projective $R G$ chain complexes, and let $f \in \operatorname{Hom}_{\mathscr{P}(R G)}\left(P_{\mathbf{.}}, Q_{.} \circ \alpha\right)$ and $g \in \operatorname{Hom}_{\mathscr{P}(R G)}\left(Q_{\mathbf{*}}, P_{\mathbf{.}}\right)$. Then

$$
\operatorname{Tr}(f \circ g)=\operatorname{Tr}(g \circ \alpha \circ f) .
$$

The Hattori-Stallings trace is also invariant up to chain homotopy.

Proposition 3.54. Let $P$. be a finitely generated projective RG-chain complex. If $f: P . \rightarrow$ $P . \circ \alpha$ and $g: P . \rightarrow P . \circ \alpha$ are chain homotopic, then $\operatorname{Tr}(f)=\operatorname{Tr}(g)$.

Proof. Let $\left\{s_{n}: P_{n} \rightarrow P_{n+1} \circ \alpha\right\}$ be a chain homotopy from $f$ to $g$. Then

$$
\begin{aligned}
\operatorname{Tr}(f)-\operatorname{Tr}(g) & =\sum_{i}(-1)^{i} \operatorname{tr}\left(f_{i}-g_{i}\right) \\
& =\sum_{i}(-1)^{i} \operatorname{tr}\left(d_{i+1} \circ \alpha \circ s_{i}+s_{i-1} \circ d_{i}\right) \\
& =\sum_{i}(-1)^{i}\left[\operatorname{tr}\left(s_{i} \circ d_{i+1}\right)+\operatorname{tr}\left(s_{i-1} \circ d_{i}\right)\right] .
\end{aligned}
$$

The last equality comes from applying commutativity. Rearranging the terms in the last sum gives $\operatorname{Tr}(f)-\operatorname{Tr}(g)=0$.

Now suppose that $C$. is an $R G$-chain complex which is chain homotopy equivalent to a finitely generated projective $R G$-chain complex. Suppose further that $\phi: C . \rightarrow C . \circ \alpha$ is a chain map. Choose a finitely generated projective $R G$-chain complex $P$., choose a chain homotopy equivalence $f: C . \rightarrow P_{.}$, and choose a lift $\psi: P_{.} \rightarrow P_{.} \circ \alpha$ of $\phi$. We get the diagram

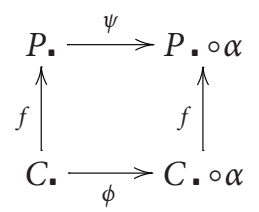

which commutes up to chain homotopy. 
Definition 3.55. The Hattori-Stallings trace of $\phi: C . \rightarrow C . \circ \alpha$ is defined to be the trace of $\psi: P . \rightarrow P_{.} \circ \alpha:$

$$
\operatorname{Tr}(\phi)=\operatorname{Tr}(\psi)
$$

We must show that $\operatorname{Tr}$ is independent of the choices we made. First, suppose that $\phi^{\prime}$ is another lift of $\phi$. Then $\psi \sim_{\text {ch }} f \circ \phi \circ f^{-1} \sim_{c h} \psi^{\prime}$ and by Proposition 3.54, $\operatorname{Tr}(\psi)=\operatorname{Tr}\left(\psi^{\prime}\right)$. Second, suppose that $Q$. is another finitely generated projective $R G$-chain complex and $g: C . \rightarrow Q$. is a chain homotopy equivalence. Then

$$
\begin{aligned}
\operatorname{Tr}\left(g \circ \phi \circ g^{-1}\right) & =\operatorname{Tr}\left(g \circ f \circ f^{-1} \circ \phi \circ f^{-1} \circ f \circ g^{-1}\right) \\
& =\operatorname{Tr}\left(f \circ g^{-1} \circ g \circ f^{-1} \circ f \circ \phi \circ f^{-1}\right) \\
& =\operatorname{Tr}\left(f \circ \phi \circ f^{-1}\right) .
\end{aligned}
$$

\section{Base-point-free Lefschetz-Nielsen invariants}

In this section, we present our base-point-free refinements of the classical geometric and algebraic Lefschetz-Nielsen invariants. We begin by defining the fundamental groupoid, and describing the way in which we think of the universal cover.

4.1. Fundamental groupoid. An important example of a groupoid is the fundamental groupoid. Let $X$ be a topological space.

Definition 4.1. The fundamental groupoid $\Pi X$ is the category whose objects are the points in $X$, whose maps are the homotopy classes rel endpoints of paths in $X$. Composition is given by concatenation of paths. To be precise, if $f$ and $g$ are paths in $X$ such that $\mathrm{f}(1)=g(0)$, then

$$
[g] \circ[f]=[f \cdot g]
$$

For each morphism, an inverse is given by traversing a representative path backwards.

This groupoid deserves to be called the fundamental groupoid since for a given point $x \in X$, the subcategory of $\Pi X$ generated by $x$ is $\pi_{1}(X, x)$. The subcategory generated by $x$ is the category with one object, $x$, and whose morphism set is $\Pi X(x, x)$. In a sense, then, the fundamental groupoid is a way of encoding in one object the fundamental groups with all possible choices of base point.

Let $f: X \rightarrow X$ be a continuous map. Then $f$ induces a functor $\Pi f: \Pi X \rightarrow \Pi X$ given by $\Pi f(x)=f(x)$ and $\Pi f(g)=f \circ g$ where $x \in X$ and $g$ is a path in $X$.

4.2. Universal cover. Let $X$ be a path connected, locally path connected, semilocally simply connected space. For each $x \in X$, one can describe the universal cover [5, page 64] of $X$ as the space

$$
\tilde{X}_{x}=(X, x)^{(I, 0)} / \sim,
$$


where $I$ is the closed unit interval and $\sim$ is the equivalence relation given by homotopy rel endpoints. The set $(X, x)^{(I, 0)}$ is given the compact-open topology, and $\tilde{X}_{x}$ is given the quotient topology. The projection map $p: \tilde{X}_{x} \rightarrow X$ is given by $p([\gamma])=\gamma(1)$.

Recall $\Pi X$, the fundamental groupoid of $X$. Let Top be the category of topological spaces.

Definition 4.2. The universal cover functor

$$
U: \Pi X \longrightarrow \text { Top }
$$

is defined by $U(x)=\tilde{X}_{x}$ for $x \in \mathrm{Ob}(\Pi X)$. For $g: x \rightarrow y$ a map in $\Pi X$, define $U(g): \tilde{X}_{x} \rightarrow$ $\tilde{X}_{y}$ by $U(g)[\gamma]=\left[g^{-1} \cdot \gamma\right]$, where $[\gamma] \in \tilde{X}_{x}$.

4.3. The geometric invariant. Fix a compact, path-connected $n$-dimensional manifold $X$ and a continuous endomorphism $f: X \rightarrow X$ such that $\operatorname{Fix}(f)$ is finite.

Let $\Pi$ be the fundamental groupoid of $X$. The map $f$ induces a functor $\varphi=\Pi f: \Pi \rightarrow$ $\Pi$ defined by $\varphi(x)=f(x)$, where $x \in \mathrm{Ob}(\Pi)$. For $g: x \rightarrow y$ a map in $\Pi$ let $\varphi(g)=f \circ g$.

Let $\operatorname{Fix}(\varphi)$ be the subcategory of $\Pi$ whose set of objects is $\operatorname{Fix}(f)$, and whose maps are the maps $g: x \rightarrow y$ in $\Pi(x, y \in \operatorname{Fix}(f))$ such that $f \circ g=g$. The category $\operatorname{Fix}(\varphi)$ decomposes into a finite number of connected components; denote them by $\mathbb{F}_{1}, \ldots, \mathbb{F}_{r}$.

Define an $\mathbb{Z} \Pi$-bimodule ${ }_{\varphi} \mathbb{Z} \Pi: \Pi \times \Pi^{\mathrm{op}} \rightarrow$ Ab given by $(x, y) \mapsto \mathbb{Z} \Pi(y, \varphi(x))$, where $x, y \in \mathrm{Ob}(\Pi)$. For $g: x \rightarrow x^{\prime}$ a map in $\Pi$ and $h: y \rightarrow y^{\prime}$ a map in $\Pi^{\mathrm{op}}$, let ${ }_{\varphi} \mathbb{Z} \Pi(g, h)=$ $\varphi(g) \circ(-) \circ h$. By definition,

$$
\begin{aligned}
H H_{0}\left(\mathbb{Z} \Pi ;{ }_{\varphi} \mathbb{Z} \Pi\right) & ={ }_{\varphi} \mathbb{Z} \Pi /\left[\mathbb{Z} \Pi,{ }_{\varphi} \mathbb{Z} \Pi\right] \\
& =\bigoplus_{x \in \mathrm{Ob}(\Pi)} \mathbb{Z} \Pi(x, \varphi(x)) / Q,
\end{aligned}
$$

where $Q$ is generated by elements of the form $\sigma-\varphi(g) \circ \sigma \circ g^{-1}$ for maps $\sigma: x \rightarrow \varphi(x)$ and $g: x \rightarrow y$ in $\Pi$.

Define

$$
\Phi:\left\{\mathbb{F}_{k}\right\}_{k=1}^{r} \longrightarrow H H_{0}\left(\mathbb{Z} \Pi ; \mathbb{Z}_{\Pi}\right)
$$

by choosing an object $x$ in $\mathbb{F}_{k}$ and mapping $\mathbb{F}_{k}$ to $\mathrm{id}_{x}: x \rightarrow x=\varphi(x)$. One can check that this is a well-defined injection.

Also, let

$$
i\left(f, \mathbb{F}_{k}\right)=\sum_{x \in \operatorname{Ob}\left(\mathbb{F}_{k}\right)} i(f, x) \in \mathbb{Z}
$$

where $i(f, x)$ is the fixed point index.

Definition 4.3. The geometric Lefschetz invariant of $f: X \rightarrow X$ is

$$
L^{\mathrm{geo}}(f)=\sum_{k} i\left(f, \mathbb{F}_{k}\right) \Phi\left(\mathbb{F}_{k}\right) \in H H_{0}(\mathbb{Z} \Pi ; \varphi \mathbb{Z} \Pi) .
$$


Theorem 4.4. The classical geometric Lefschetz invariant and the base-point-free geometric Lefschetz invariant correspond under an isomorphism

$$
A: \mathbb{Z} \pi_{\phi} \longrightarrow H H_{0}\left(\mathbb{Z} \Pi ;{ }_{\varphi} \mathbb{Z}\right)
$$

The isomorphism $A$ is not canonical; it depends on choosing a path from $*$ to $f(*)$. On the other hand, $\mathrm{HH}_{0}\left(\mathbb{Z} \Pi ; \mathbb{Z}_{\varphi} \mathbb{Z}\right)$ is canonical.

Proof. Recall that in the classical definition, we have chosen a base point $*$ and a base path $\tau$. The fundamental group $\pi_{1}(X, *)$ is denoted by $\pi$, the map on $\pi$ induced by $f: X \rightarrow X$ and the base path $\tau$ is denoted by $\phi$, and the injection $\left\{F_{i}\right\}_{i=1}^{s} \rightarrow \pi_{\phi}$ is denoted by $\Phi$.

Step 1. After appropriate reordering of the fixed point classes $F_{1}, \ldots, F_{s}, s=r$ and $F_{i}=$ $\mathrm{Ob}\left(\mathbb{F}_{i}\right)$. This can be seen as follows. If $x$ and $y$ are equivalent in $\operatorname{Fix}(f)$, then there exists a path $v$ from $x$ to $y$ in $X$ such that $\nu \cdot(f \circ v)^{-1} \simeq *$. But this is equivalent to saying that $\nu$ is a map in $\operatorname{Fix}(\varphi)$ from $x$ to $y$, and hence that $x$ and $y$ are in the same connected component of $\operatorname{Fix}(\varphi)$.

Step 2. Define an isomorphism of abelian groups

$$
A: \mathbb{Z} \pi_{\phi} \longrightarrow H H_{0}\left(\mathbb{Z} G ;{ }_{\varphi} \mathbb{Z} G\right)
$$

by $A(\omega)=\omega \cdot \tau=\tau \circ \omega$, where $[\omega] \in \pi$.

To see that $A$ is well defined, suppose that $[\omega]$ and $\left[\omega_{1}\right]$ are equivalent in $\mathbb{Z} \pi_{\phi}$. By definition, there exists $g \in \pi$ such that $\omega_{1}=g \cdot \omega \cdot \tau \cdot(f \circ g)^{-1} \cdot \tau^{-1}$. Hence, $\tau \circ \omega_{1}=$ $\varphi\left(g^{-1}\right) \circ \tau \circ \omega \circ g=\tau \circ g$ in $H H_{0}(\mathbb{Z} G ; \varphi \mathbb{Z} G)$, and $A$ is well-defined.

To see that $A$ is an epimorphism, suppose that $\sigma: x \rightarrow \varphi(x) \in H H_{0}\left(\mathbb{Z} G ;{ }_{\varphi} \mathbb{Z} G\right)$. Choose a path $\mu$ in $X$ from $*$ to $x$, that is, a map $\mu: * \rightarrow x$ in $G$. Then $\sigma=\varphi\left(\mu^{-1}\right) \circ \sigma \circ \mu$ in $H H_{0}\left(\mathbb{Z} G ; \varphi^{Z} G\right)$, and $\mu \cdot \sigma \cdot(f \circ \mu)^{-1} \cdot \tau^{-1}$ gives an element in $\pi$ which is mapped to $\sigma$ by A.

The last thing to check is that $A$ is a monomorphism. Suppose $[\omega]$ and $\left[\omega_{1}\right]$ are elements of $\pi$ such that $\tau \circ \omega=\tau \circ \omega_{1}$. Then there exists $g \in \mathrm{Ob}(G)$ such that $\tau \circ \omega_{1}=$ $\varphi\left(g^{-1}\right) \circ \tau \circ \omega \circ g$. It follows that $\omega_{1}=g \cdot \omega \cdot \tau \cdot(f \circ g)^{-1} \cdot \tau^{-1}$ and hence that $\left[\omega_{1}\right]$ is equivalent to $[\omega]$ in $\mathbb{Z} \pi_{\phi}$.

Step 3. Let $F$ be a fixed point class, and $\mathbb{F}$ the corresponding connected component of $\operatorname{Fix}(\varphi)$. For any choice of $x \in F$ and path $\mu$ from $*$ to $x$, we have that $A(\Phi(F))=A(\mu$. $\left.(f \circ \mu)^{-1} \cdot \tau^{-1}\right)=\varphi\left(\mu^{-1}\right) \circ \mu=\operatorname{id}_{x}$ in $H H_{0}(\mathbb{Z} G ; \varphi \mathbb{Z} G)$.

Therefore, the image of

$$
L^{\mathrm{geo}}(f, *, \tau)=\sum_{k=1}^{s} i\left(f, F_{k}\right) \Phi\left(F_{k}\right) \in \mathbb{Z} \pi_{\phi}
$$

is equivalent to

$$
L^{\text {geo }}(f)=\sum_{k=1}^{r} i\left(f, \mathbb{F}_{k}\right) \Phi\left(\mathbb{F}_{k}\right) \in H H_{0}\left(\mathbb{Z} G ;{ }_{\varphi} \mathbb{Z} G\right) .
$$


4.4. The algebraic invariant. Let $X$ be a finite CW complex and $f: X \rightarrow X$ a continuous map. Let $\Pi=\Pi X$ be the fundamental groupoid of $X$ and let $\varphi: \Pi \rightarrow \Pi$ be the functor induced by $f$, as above.

The map $f$ induces a natural transformation $\tilde{f}: U \rightarrow U \circ \varphi$. Given an object $x$ in $\Pi$, $\tilde{f}_{x}: \tilde{X}_{x} \rightarrow \tilde{X}_{f(x)}$ is defined by $[\gamma] \mapsto[f \circ \gamma]$, where $[\gamma] \in \tilde{X}_{x}$. One can check naturality.

There is a functor $S:$ Top $\rightarrow \mathrm{Ch}(\mathbb{Z})$ given by taking the singular chain complex of a space. If $f: X \rightarrow Y$ is a continuous map, then $S(f): S(X) \rightarrow S(Y)$ is given by $\sigma \mapsto f \circ \sigma$, where $\sigma: \Delta^{n} \rightarrow X$. Here, $\Delta^{n}$ is the standard $n$-simplex.

Let $C$. be the $\mathbb{Z} \Pi$-chain complex given by the composition

$$
\Pi \stackrel{U}{\longrightarrow} \operatorname{Top} \stackrel{S}{\longrightarrow} \mathrm{Ch}(\mathbb{Z})
$$

The map $f$ induces a natural transformation $\widetilde{f_{*}}: S U \rightarrow S U \varphi$. Given an object $x$ in $\Pi$, let $\widetilde{f}_{*}(x): S\left(\tilde{X}_{x}\right) \rightarrow S\left(\tilde{X}_{f(x)}\right)$ be given by $\sigma \mapsto \tilde{f}_{x} \circ \sigma$, where $\sigma: \Delta^{n} \rightarrow \tilde{X}_{x}$. Naturality of $\tilde{f}_{*}$ follows from naturality of $\tilde{f}$. Hence, $\tilde{f}_{*}$ is a $\varphi$-linear chain map C. $\rightarrow$ C. As usual, $\tilde{f}_{*}$ is given by a family of $\varphi$-linear natural transformations $\tilde{f}_{n}: C_{n} \rightarrow C_{n}$.

The singular chain complex of a finite CW complex is chain homotopy equivalent to a finitely generated projective $\mathbb{Z} \Pi$ chain complex. Hence, the Hattori-Stallings trace of $\tilde{f}_{*}$ is defined, and we can define the algebraic Lefschetz invariant as follows.

Definition 4.5. The algebraic Lefschetz invariant of $f: X \rightarrow X$ is

$$
L^{\mathrm{alg}}(f)=\operatorname{Tr}\left(\tilde{f}_{*}\right)=\sum_{k \geq 0}(-1)^{k} \operatorname{tr}\left(\tilde{f}_{k}\right) \in H H_{0}(\mathbb{Z} \Pi ; \varphi \mathbb{Z} \Pi)
$$

As an immediate corollary of Proposition 3.51 we get the following theorem.

Theorem 4.6. The classical algebraic Lefschetz invariant and the base point free algebraic Lefschetz invariant correspond under the isomorphism

$$
A: \mathbb{Z} \pi_{\phi} \longrightarrow H H_{0}\left(\mathbb{Z} \Pi ;{ }_{\varphi} \mathbb{Z} \Pi\right)
$$

\section{References}

[1] H. Bass, Euler characteristics and characters of discrete groups, Inventiones Mathematicae 35 (1976), no. 1, 155-196.

[2] __ Traces and Euler characteristics, Homological Group Theory (Proc. Sympos., Durham, 1977), London Math. Soc. Lecture Note Ser., vol. 36, Cambridge University Press, Cambridge, 1979, pp. 1-26.

[3] R. F. Brown, The Lefschetz Fixed Point Theorem, Scott, Foresman, Illinois, 1971.

[4] R. Geoghegan, Nielsen fixed point theory, Handbook of Geometric Topology, North-Holland, Amsterdam, 2002, pp. 499-521.

[5] A. Hatcher, Algebraic Topology, Cambridge University Press, Cambridge, 2002.

[6] B. J. Jiang, Lectures on Nielsen Fixed Point Theory, Contemporary Mathematics, vol. 14, American Mathematical Society, Rhode Island, 1983.

[7] W. Lück, Transformation Groups and Algebraic K-Theory, Lecture Notes in Mathematics, vol. 1408, Mathematica Gottingensis, Springer, Berlin, 1989. 
20 A base-point-free definition of the Lefschetz invariant

[8] - The universal functorial Lefschetz invariant, Fundamenta Mathematicae 161 (1999), no. $1-2,167-215$.

[9] J. Stallings, Centerless groups-an algebraic formulation of Gottlieb's theorem, Topology. An International Journal of Mathematics 4 (1965), no. 2, 129-134.

Vesta Coufal: Department of Mathematics, Fort Lewis College, Durango, CO 81301, USA

E-mail address: coufal_v@fortlewis.edu 